\title{
Intercepting Virtual Ball in Immersive Virtual Environment
}

\author{
Massimiliano Valente, Davide Sobrero, Andrea Brogni, and Darwin Caldwell \\ Advanced Robotics Dept. - Istituto Italiano di Tecnologia, Genoa, Italy \\ \{massimiliano.valente, davide.sobrero, andrea.brogni, \\ darwin.caldwell\}@it.it
}

\begin{abstract}
Catching a flying ball is a difficult task that requires sensory systems to calculate the precise trajectory of the ball to predict its movement, and the motor systems to drive the hand in the right place at the right time.

In this paper we have analyzed the human performance in an intercepting task performed in an immersive virtual environment and the possible improvement of the performance by adding some feedback.

Virtual balls were launched from a distance of $11 \mathrm{~m}$ with 12 trajectories. The volunteers was equipped only with shutter glasses and one maker on backhand to avoid any constriction of natural movements. We ran the experiment in a natural scene, either without feedback or with acoustic feedback to report a corrects intercept. Analysis of performance shows a significant increment of successful trials in feedback condition. Experiment results are better with respect to similar experiment described in literature, but performances are still lower to results in real world.
\end{abstract}

Keywords: Virtual Reality, Ecological Validity, Interceptive Action.

\section{Introduction}

In real life, interaction with different objects is driven by a set of sensory stimuli, first of all visual and haptic ones: if we lose some of them our performances decrease. When we want to interact with a moving object, we need to know its physical characteristics and its space displacement so that our movements toward the target are well directed.

In particular, catching a flying ball is a difficult task that requires our sensory system to calculate the precise trajectory of the ball to predict its movement, and our motor system to drive the hand in the right place at the right time.

Such characteristics set makes this task suitable for a good evaluation of human performances in terms of precision, immersion and adaptation in virtual reality.

The aim of our study is to design a more natural way of interaction and, thus, to evaluate if the performance increases and the goal-directed movement is more accurate, and overall to evaluate the human reactions in a virtual environment.

In our experiment, we expect that the natural wave to reach a flying ball produces better performance with respect to the same task performed with other handed devices. Thus, besides the performance results, we have also evaluated the characteristics of the hand movement, to certify the similarity of the trajectory with respect to the same task in a real environment. 
The remind of the paper is organized as follows: in Sec. 2 we outline some relevant related work in literature; in Sec. 3 we describe the design of experiment; in Sec. 4 we present the results obtained from the subjects performing the experiment and in Sec.5 we summarize the most relevant results and discuss some possible future works.

\section{Related Works}

The spatial perception is important variables in our task, we must consider the potential causes of different perception between real-word and virtual environment like graphic characteristics and differences between natural vision and perception in virtual environment: for more details, see Murgia and Sharkey [10]. In particular, distance perception is influenced by these perceptive differences. Different previous works report that object's distance from observer is underestimated more in virtual environment than in real [114].

Literature on reaching ball in physical world is divided in three research areas: the outfielder problem, estimation of reaching and catching fly ball.

The outfielder problem regards the movement to position of ball's fall [5]9]; the test of the outfielder problem is translated by Fink et. al in virtual environment with use of HMD [7]. Experiments of estimation of reaching regard the indication of balls positions before or after balls passages [12]. Catching fly ball studies analyze the trajectory perception in binocular and monocular conditions [8], the performance with several velocity [13], the performance with several angle trajectories [4].

The literature on catching or intercepting balls in immersive virtual reality is addressed on all body movement's analysis sport performance both wit HMD [7] and in CAVE [2|3|6].

Zaal and Michaels [15] studied the judging of trajectories and intercepting movements for intercepting task in CAVE, they also made analysis of intercepting performances but their results show that the volunteers intercepted only the $15 \%$ of balls. They designed their experiment without any environment and they captured hand movements with a wand then the volunteers held. They used only throws to subjects bound without different angles of approach.

\section{The Experiment}

We plan our experiment starting from a typical training task for baseball players: to catch a ball thrown from an automatic system. We designed a simple virtual environment where the subject can perform a similar repetitive task.

\subsection{System Setup}

We carried out our experiment in an immersive virtual environment system: the room was equipped with two stereographic projectors Christie Mirage $\mathrm{S}+3 \mathrm{~K}$ that allow visualizing a wide scene on a screen of $4 \mathrm{~m}$ of length and $2 \mathrm{~m}$ of height. The system is integrated with the IS900 from Intersense: a 6-DOF system for wide area tracking; we used it to track the position of the head of the subject. 
We also used the Optitrack FLEX:V100r2 motion capture system, with infrared cameras to track the subject hand with a passive marker fixed on his right back hand.

The graphical aspect of the experiment was implemented using the XVR 1 framework for developing virtual reality applications. We used in addiction a physic simulation engine (Physx $\sqrt[2]{2}$ ) to calculate in real time the ball trajectory as real as possible.

\subsection{Procedure and Design}

The virtual environment was composed of a green grass with on a wooden board $11 \mathrm{~m}$ away from the observer. We placed of the board every $2 \mathrm{~m}$ three black point, to indicate the different sources on the throws. A picture of the starting setup of the experiment is shown in Fig. 1

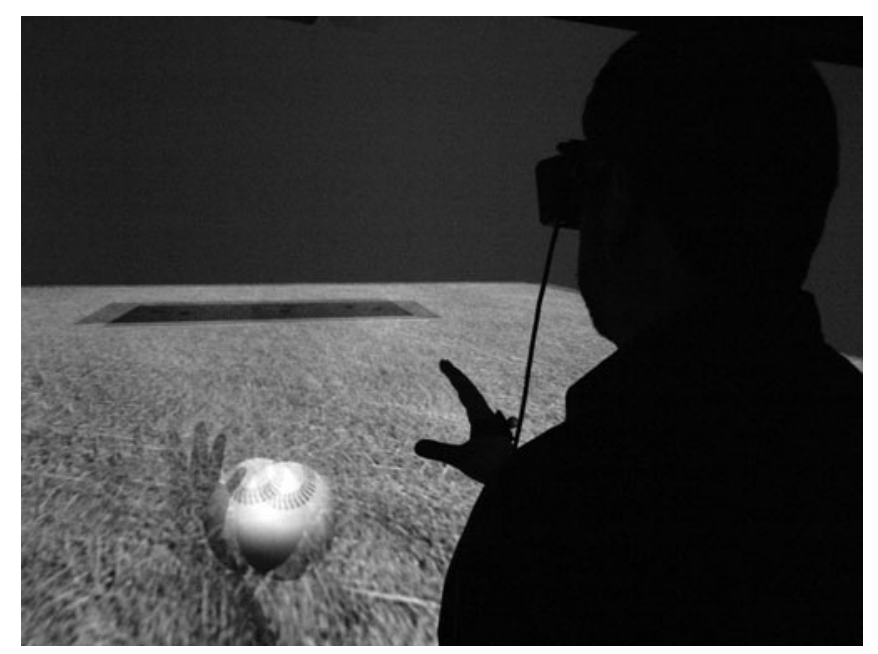

Fig. 1. The initial setup: the ball is in front of the subject

At the beginning of the experiment, the volunteer stood in front of the screen with his feet on a blue line posed one meter away from the screen. A virtual softball ball, of $10 \mathrm{~cm}$ diameter, was placed between the screen and the subject, at subject's chest height, to make clear the actual dimension of the ball.

During the experimental sessions, the subjects were asked to try to intercept the virtual softball ball with the right hand. In every trial, the ball was thrown with constant initial speed, about $10 \mathrm{~m} / \mathrm{s}$, from one of the three black points randomly. The ball could have three different elevations from the floor (high $38.5^{\circ}$, central $37^{\circ}$ and low $35.5^{\circ}$ ) and four different angles respects to the subject (azimuth), as explained in Table 1.

We designed the trajectories through these parameters to obtain four classes of arrival area: L-balls for balls arriving on the left of the subject, C-balls for ball arriving in

\footnotetext{
${ }^{1}$ www.vrmedia.it

2 www.nvidia.com
} 
Table 1. Different azimuth angles for trajectory

\begin{tabular}{rcccc}
\hline & \multicolumn{4}{c}{ Azimuth } \\
\cline { 2 - 5 } & L-balls & C-balls & R-balls & RR-balls \\
\hline Left hole & $9.3^{\circ}$ & $10.3^{\circ}$ & $11.3^{\circ}$ & $12.3^{\circ}$ \\
Central hole & $-1.0^{\circ}$ & $0.0^{\circ}$ & $1.0^{\circ}$ & $2.0^{\circ}$ \\
Right hole & $-11.3^{\circ}$ & $-10.3^{\circ}$ & $-9.3^{\circ}$ & $-8.3^{\circ}$ \\
\hline
\end{tabular}

front of the subject, R-balls for balls arriving on the right and RR-balls for the ones arriving on the extreme right, still reachable from the hand subject. In Fig. 2 we show the idealized target position.

We tried with some volunteers trajectories arriving to the extreme left, but this throws came out hard to intercept because the balls was out of range of the right hand.

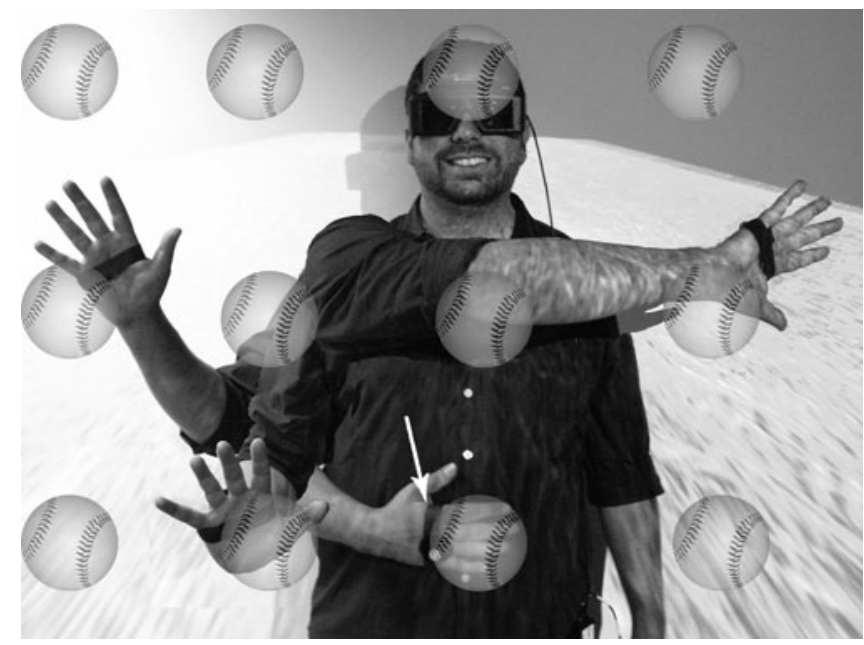

Fig. 2. Idealized target position is indicated by balls. The arrow shows the hand in start position and the marker on back hand.

The subject was asked to stay in fixed position, its feet on the blue line and the right hand on its navel, in front of the screen, before the ball starts and to move the right hand to intercept the ball as quickly as possible when the ball starts to move.

To reach the ball far from him, he could move the body, but he could not move his feet.

The experiment was made up of five sessions: one training session with ten throws and four experimental sessions of ninety throws each, for a total of 370 trials for subject. 


\subsection{Participants}

We performed the experiment with twenty volunteers (thirteen men and seven women) between 25 and 32 years of age, with height between $1.65 \mathrm{~m}$ and $1.80 \mathrm{~m}$. All the participants reported normal or correct to normal vision. All the subjects right handed, as checked with the Edinburgh Handedness Inventory [11].

The volunteers reported nothing or little previous virtual reality experience. Five people reported experience in ball games such as basketball, volleyball or tennis and all men reported experience in soccer (but not as goalkeeper).

The volunteers participated in to this experiment after giving informed consent.

We divided the volunteers into two random group of ten people. The first group did not receive any kind of feedback, either visual or other, for rights intercepting ball during the experiment (NoFeedback Group). The second group had sounds feedbacks, one for rights catching ball and one for fail, during the experiment (Feedback Group). Moreover, at the end of every session the subjects of this group were informed of their percentage of success.

\section{Results}

We recorded data from the head sensor for the head movements, the position of the hand during the ball throwing and the position of the ball during the fly.

We have examined subjects catching behavior and the immersion in virtual environment degree. We analyzed the percentage of successful catches and we compared results of NoFeedback Group with results of Feedback Group.

In addiction we analyzed the hand movement characteristics (peak of maximum velocity, latency before start the movement and time to catching) to verify if our results can be comparable with the same data found in experiments in real word showed in literature.

\subsection{Catching Performance}

To assess the success in the catching of the balls, we calculated at each time step the distance between the marker placed on subject's hand and the center of the virtual ball, when the ball was in front of the hand and not behind.

We assessed a successful catch (hit) when this distance was less than $10 \mathrm{~cm}$. We calculated the global hit probability for both groups of subjects, the hit probability for all trajectories and hit probability for the 12 intercept areas. A summary of the performances for all intercept areas can be found on Table 2

We can see that the performance without acoustic feedback is significantly lower than performances helped by a feedback. The average of performance of No feedback Group was 0.53 with a standard error of 0.014, the performance of Feedback Group was 0.61 with standard error of $0.012(p<0.001)$.

The graphs in Fig. 3 show mean and standard error for all types of throw. We can observe that the performances of Feedback group are always better than performances of NoFeedback group for throws from left hole (hit probability: 0.61 vs. 0.5 ) and central 
Table 2. Results

\begin{tabular}{crrrrrrrrcc}
\hline & \multicolumn{4}{c}{ L } & \multicolumn{2}{c}{ C } & \multicolumn{2}{c}{ R } & \multicolumn{2}{c}{ RR } \\
\cline { 2 - 10 } & & Mean & SEM & Mean & SEM & Mean & SEM & Mean & SEM \\
\cline { 2 - 10 } High & $F b$ & 0.557 & 0.045 & 0.637 & 0.041 & 0.651 & 0.035 & 0.581 & 0.053 \\
& $N o F b$ & 0.488 & 0.044 & 0.599 & 0.047 & 0.568 & 0.049 & 0.489 & 0.039 \\
\hline \multirow{2}{*}{ Central } & $F b$ & 0.657 & 0.038 & 0.740 & 0.030 & 0.742 & 0.029 & 0.612 & 0.041 \\
& $N o F b$ & 0.567 & 0.061 & 0.632 & 0.039 & 0.644 & 0.036 & 0.567 & 0.050 \\
\hline \multirow{2}{*}{ Low } & $F b$ & 0,443 & 0,041 & 0.586 & 0.034 & 0.569 & 0.036 & 0.520 & 0.036 \\
& $N o F b$ & 0.392 & 0.055 & 0.409 & 0.058 & 0.536 & 0.045 & 0.468 & 0.044 \\
\hline
\end{tabular}

hole (hit probability: 0.64 vs. 0.53 ). This difference is not present for throws from right hole: the NoFeedback group's performances reach the Feedback group's performances (hit probability: 0.57 vs. 0.57 ).

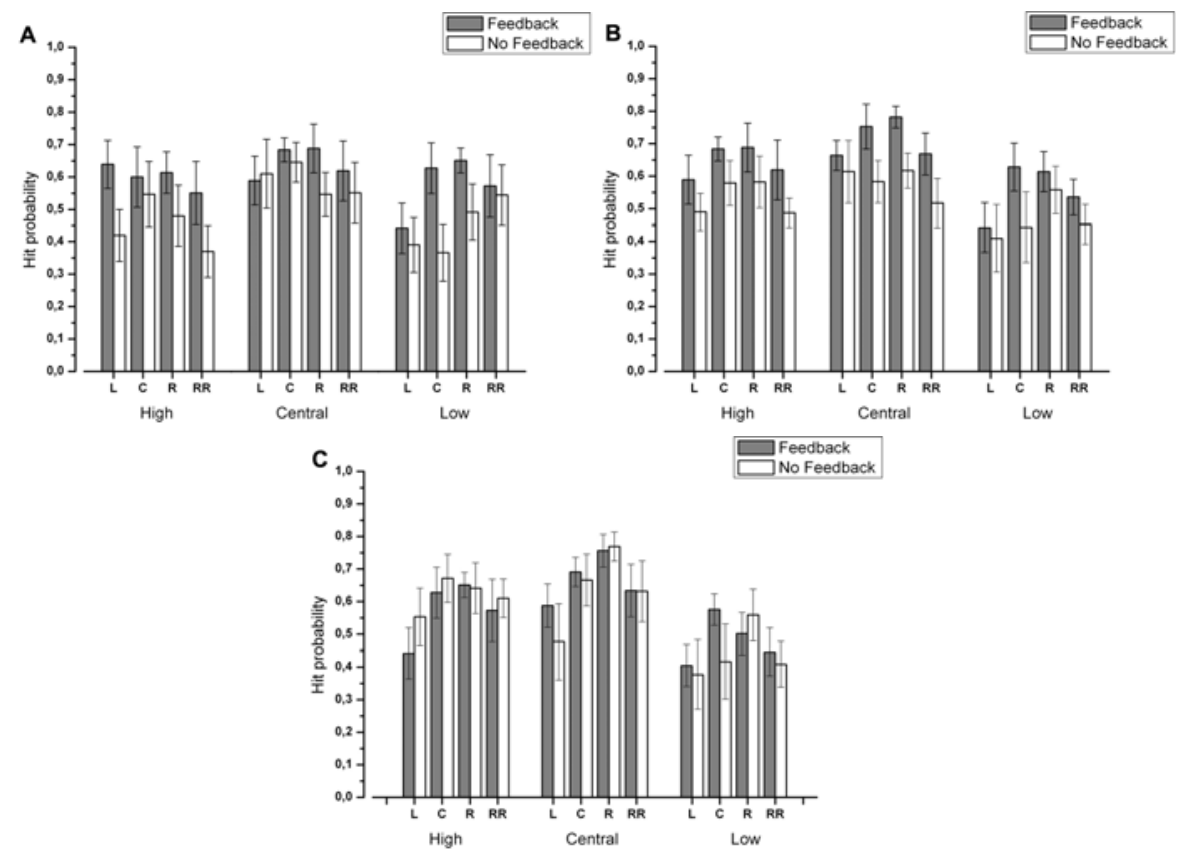

Fig. 3. Hit success percentage of all throws. (A) throws from right hole. (B) throws from central hole. (C) throws from right hole.

ANOVA analysis shows significant effect of the elevation factor in both groups.

For the NoFeedback group the effect is significant between $38.5^{\circ}$ and $35.5^{\circ}$ with $p=0.017$ and between $37^{\circ}$ and $35.5^{\circ}$ with $p>0.001$. The difference is not significant between $38.5^{\circ}$ and $37^{\circ}(p=0.08)$. 


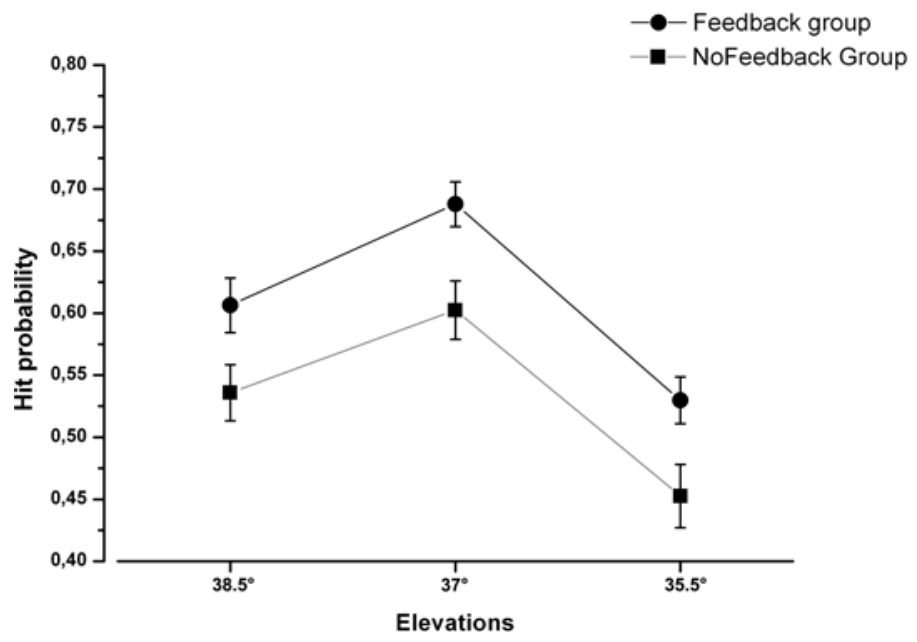

Fig. 4. Hit probability for different elevations

For the Feedback group the effect is significant for all elevation among them $\left(38.5^{\circ}\right.$ vs. $37^{\circ} p=0.001 ; 38.5^{\circ}$ vs. $35.5^{\circ} p=0.003 ; 37^{\circ}$ vs. $\left.35.5^{\circ} p>0.001\right)$.

Feedback's effect is show in Fig. 4, this effect is significant for all elevations always with $p<0.001$.

The analysis performances reveals a good interpretation of balls' trajectories but the intercept performances are worse than results made in real environment, that record performances around $90 \%$ of hit [813] versus $74 \%$ of our better mean performance.

However our hit probabilities are better than the performance indicated by Zaal and Michaels [15] for the same type of throws in virtual environment.

\subsection{Hand Movement}

Latency. We calculated the latencies from the instant in which the ball starts, to the moment when movement speed is about $5 \mathrm{~m} / \mathrm{s}$. Mean latency is $417 \mathrm{~ms}$; this results is consistent with the acquisition in real task [8].

There are some differences in latencies between the different types of throws. Latencies times are inverse proportional to distances between the start position and the probable impact point, and they have correlation with left or right side of catch. Post hoc analysis shows significant differences between latencies for L-balls and R-balls. Graph of the mean latencies grouped for area of intercept is shown in Fig. 5 a.

Maximal Velocity Analysis. The means of maximal velocity for the various trajectories (Fig. 5b) is the mirror image of latencies graph: minor latency is equal to major speed movement to left side.

Heuristics Observations. We made two particular observation on subject's behavior during the task execution. 

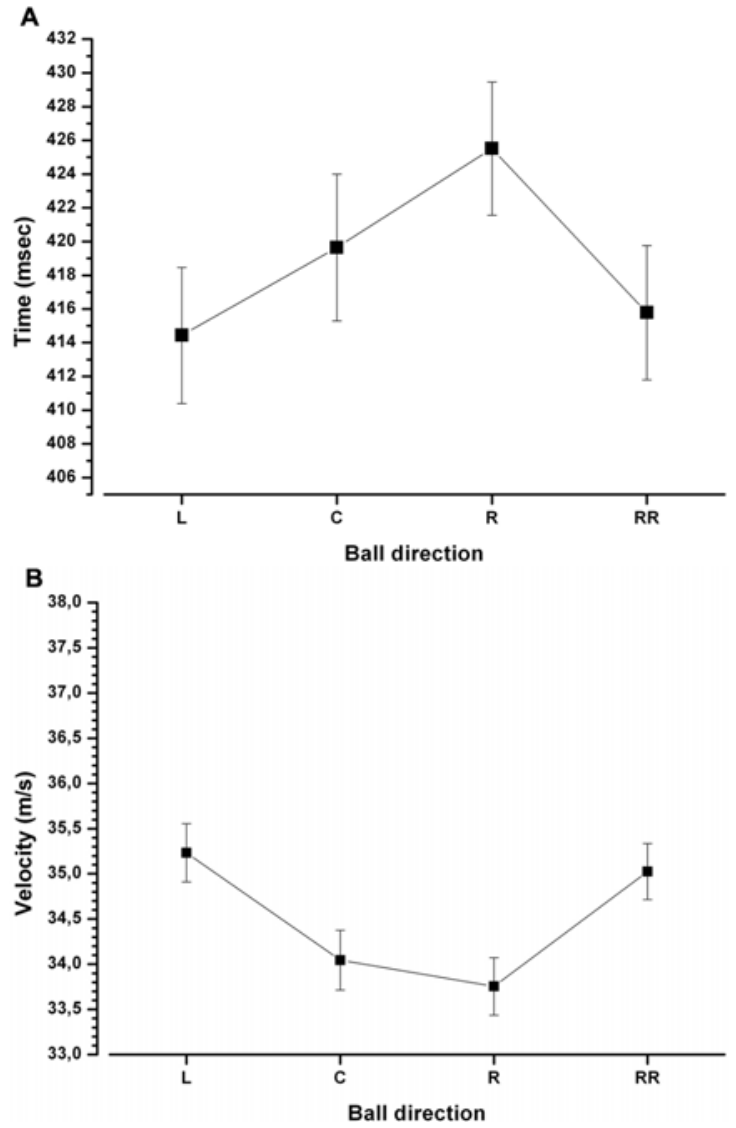

Fig. 5. (A) Mean latencies of hand movement for area of intercept. (B) Maximal velocity of hand for area of intercept.

We observed that many volunteers made an hand's grasping movement on virtual balls during the experiment. This behavior lasted about two or three sessions (including training session) and after only few people carried on to close the hand.

Moreover, the subjects had to be still with feet during the experimental sessions, according to instructions, but they had no instructions for rest of body. Few volunteers started immediately to move the body to reach the balls, most people started to move the body only after two or three sessions.

\section{Conclusions}

The experiment gave us positive results with respect to previous experiences: the possibility to perform a natural reaching movement without using any handed device and the ecologic setup have raised the performances of the subjects beyond the results present in literature, in spite of the presence of a major number of trajectories. 
We have shown that by giving in real time an acoustic feedback the performance increases, allowing a correction of the eye/hand coordination and, probably, giving a reward element absent in the case without feedback.

The fact that in the case of throws from the right hole the feedback does not give any aid is probably due to the more clear visibility of this types of trajectories.

Nevertheless, we do not reach the performances expected in the real case: this can be caused by perceptive problems mentioned in Sec. 2, or from the relative lack of proposal feedbacks.

We are working on different types of feedback and combination of more than one feedback at the same time, to verify if the performances can be further improved.

\section{References}

1. Armbrster, C., Wolter, M., Kuhlen, T., Spijkers, W., Fimm, B.: Depth perception in virtual reality: Distance estimations in peri- and extrapersonal space. Cyber Psychology \& Behavior 11(1), 9-15 (2008)

2. Bideau, B., Kulpa, R., Vignais, N., Brault, S., Multon, F., Craig, C.: Using virtual reality to analyze sports performance. IEEE Computer Graphics and Applications 30(2), 14-21 (2010)

3. Bideau, B., Multon, F., Kulpa, R., Fradet, L., Arnaldi, B., Delamarche, P.: Using virtual reality to analyze links between handball thrower kinematics and goalkeeper's reactions. Neuroscience Letters 372(1-2), 119-122 (2004)

4. Bockemhl, T., Troje, N.F., Drr, V.: Inter-joint coupling and joint angle synergies of human catching movements. Human Movement Science 29(1), 73-93 (2010)

5. Chapman, S.: Catching a baseball. American Journal of Physics 36(10), 868-870 (1968)

6. Craig, C.M., Goulon, C., Berton, E., Rao, G., Fernandez, L., Bootsma, R.J.: Optic variables used to judge future ball arrival position in expert and novice soccer players. Attention, Perception, \& Psychophysics 71(3), 515-522 (2009)

7. Fink, P.W., Foo, P.S., Warren, W.H.: Catching fly balls in virtual reality: A critical test of the outfielder problem. Journal of Vision 9(13), 1-8 (2009)

8. Mazyn, L.I.N., Lenoir, M., Montagne, G., Savelsbergh, G.J.P.: The contribution of stereo vision to one-handed catching. Experimental Brain Research 157(3), 383-390 (2004)

9. McLeod, P., Dienes, Z.: Do fielders know where to go to catch the ball or only how to get there? Journal of Experimental Psychology: Human Perception and Performance 22(3), 531543 (1996)

10. Murgia, A., Sharkey, P.M.: Estimation of distances in virtual environments using size constancy. The International Journal of Virtual Reality 8(1), 67-74 (2009)

11. Oldfield, R.C.: The assessment and analysis of handedness: The edinburgh inventory. Neuropsychologia 9(1), 97-113 (1971)

12. Peper, L., Bootsma, R.J., Mestre, D.R., Bakker, F.C.: Catching balls: How to get the hand to the right place at the right time. Journal of Experimental Psychology: Human Perception and Performance 20(3), 591-612 (1994)

13. Tijtgat, P., Bennett, S., Savelsbergh, G., De Clercq, D., Lenoir, M.: Advance knowledge effects on kinematics of one-handed catching. Experimental Brain Research 201(4), 875884 (2010), 10.1007/s00221-009-2102-0

14. Wann, J.P., Rushton, S., Mon-Williams, M.: Natural problems for stereoscopic depth perception in virtual environments. Vision Research 35(19), 2731-2736 (1995)

15. Zaal, F.T.J.M., Michaels, C.F.: The information for catching fly balls: Judging and intercepting virtual balls in a cave. Journal of Experimental Psychology: Human Perception and Performance 29(3), 537-555 (2003) 